\title{
A Rare Case of Chronic Expanding Intrapericardial Hematoma with Refractory Right-sided Heart Failure 30 Years after the Surgical Repair of Tetralogy of Fallot
}

\author{
Satoshi Nakawatase ${ }^{1}$, Yoshinori Asae ${ }^{1}$, Yoshiyuki Miyamoto ${ }^{1}$, Masaoki Miyamoto', \\ Yasushi Okumoto $^{1}$, Hiroki Mizoguchi ${ }^{2}$, Masayuki Sakaki ${ }^{2}$ and Keizo Kimura ${ }^{1}$
}

\begin{abstract}
:
We herein report the case of a 79-year-old man who presented with right-sided heart failure (HF) 27 years after undergoing surgery for tetralogy of Fallot. The HF did not respond well to oral diuretics. Transthoracic echocardiography and chest X-ray failed to determine the cause of the HF for three years. An intrapericardial mass located just behind the sternum, was finally identified on computed tomography. The mass had compressed the right ventricle, causing right-sided HF. Pre-surgical diagnostic images led to suspicion of a chronic expanding intrapericardial hematoma (CEIH), and the CEIH was surgically removed. The patient's symptoms improved markedly.
\end{abstract}

Key words: chronic expanding hematoma, right-sided heart failure, tetralogy of Fallot, intrapericardial cavity, open-heart surgery, diastolic dysfunction

(Intern Med 60: 2627-2631, 2021)

(DOI: 10.2169/internalmedicine.6800-20)

\section{Introduction}

The first chronic expanding hematoma (CEH) was described by Reid et al. in 1980 (1). Hematomas usually resolve rapidly without clinical problems. However, CEH persists and continues to develop for over a month after the initial injury. They can grow to form space-occupying lesions, which can evoke symptoms. After heart injuries, such as those caused by surgery (2), trauma (3), or epicardial injuries (4), hematomas can develop in the pericardial space. Such hematomas are called chronic expanding intrapericardial hematomas (CEIHs). In open-heart surgery, hematomas usually develop and produce symptoms within a few days, but CEIHs are very rare.

We herein report a case in which a CEIH caused refractory right-sided heart failure (HF) 30 years after the repair of tetralogy of Fallot (TOF). The CEIH was successfully surgically treated.

\section{Case Report}

A 79-year-old man was admitted to our hospital with refractory right-sided HF. He had undergone surgery for TOF 30 years earlier at another hospital. He had been taking oral anticoagulants for atrial fibrillation for over 10 years. He had been suffering from edema in his legs for three years before his admission. Although serial transthoracic echocardiography (TTE), chest X-ray (CXR), and blood examinations were performed, the reason for the refractory edema was unclear. The edema was initially ameliorated by administering oral diuretics, but it recurred and gradually worsened.

On admission, the patient's blood pressure was 142/49 $\mathrm{mmHg}$, and his pulse rate was 57 beats/min and irregular. CXR showed cardiac enlargement (cardiothoracic ratio: $58 \%$ ) and right pleural effusion (Fig. 1A). An electrocardiogram revealed atrial fibrillation and a heart rate of 64 beats/ min. TTE demonstrated a preserved left ventricular (LV) systolic function with a LV diastolic dimension of $51 \mathrm{~mm}$

${ }^{1}$ Division of Cardiology, Heart Center, Kinan Hospital, Japan and ${ }^{2}$ Division of Cardiovascular Surgery, Heart Center, Kinan Hospital, Japan Received: November 27, 2020; Accepted: January 6, 2021; Advance Publication by J-STAGE: March 8, 2021 Correspondence to Dr. Keizo Kimura, k0301crm@wakayama-med.ac.jp 


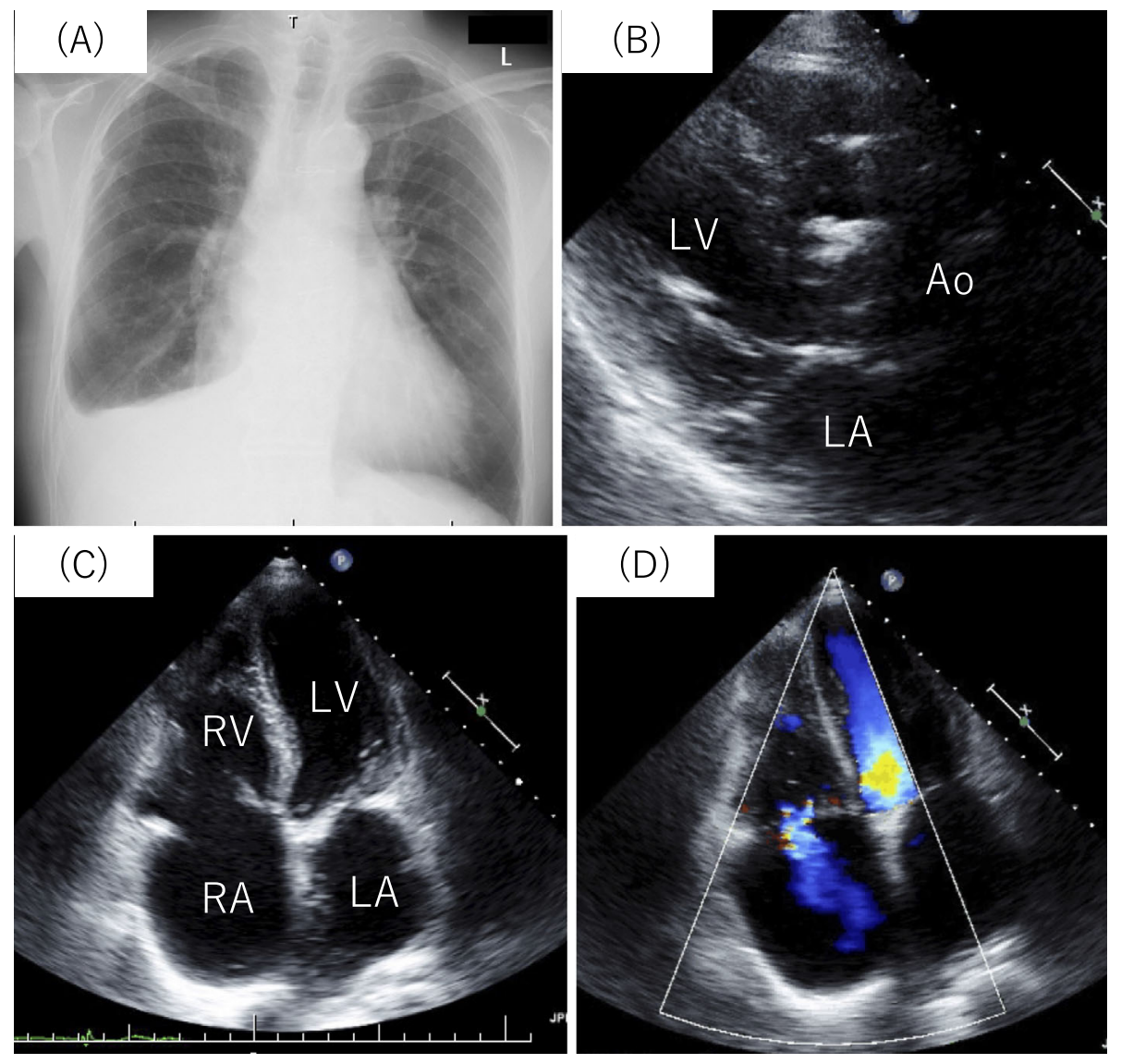

Figure 1. Chest X-ray and transthoracic echocardiography findings. (A) Chest X-ray showed right pleural effusion but no abnormal masses. No abnormal intrapericardial mass was seen on the parasternal long-axis view (B) or apical 4-chamber view (C) of transthoracic echocardiography. (D) Tricuspid regurgitation with a flow velocity of $2.74 \mathrm{~m} / \mathrm{s}$ was observed. Ao: aorta, RA: right atrium, RV: right ventricle, $L A$ : left atrium, LV: left ventricle

and a LV systolic dimension of $33 \mathrm{~mm}$ (Fig. 1B). The right atrium was moderately enlarged (Fig. 1C). The tricuspid annular plane systolic excursion had decreased to $9 \mathrm{~mm}$ (normal range: $\geq 17 \mathrm{~mm}$ ), and tricuspid regurgitation was observed along with a regurgitant flow velocity of $2.74 \mathrm{~m} / \mathrm{s}$ (Fig. 1D). No abnormal masses were identified around the heart on TTE.

Contrast-enhanced chest computed tomography (CT) demonstrated a mass with a diameter of $53 \mathrm{~mm} \times 37 \mathrm{~mm}$. The mass was surrounded by a calcified capsule and was located just behind the sternum, compressing the right ventricle (RV) (Fig. 2). It extended to the anterior walls of the ascending aorta and main pulmonary artery. On magnetic resonance imaging (MRI), T1-weighted images showed a highintensity area surrounded by a low-intensity rim, whereas $\mathrm{T}$ 2-weighted images demonstrated a mixture of nonhomogeneous low- and high-intensity areas surrounded by a low-intensity rim, suggesting that the mass was a hematoma (Fig. 3). Cardiac catheterization revealed a mean pulmonary capillary wedge pressure (PCWP) of $21 \mathrm{mmHg}$, a pulmonary artery pressure of 37/16 (mean: 25) $\mathrm{mmHg}$, a mean right atrial pressure of $21 \mathrm{mmHg}$ with a rapid and deep y descent, and a right ventricular end-diastolic pressure of 15 $\mathrm{mmHg}$ together with an abnormal diastolic filling pattern
(Fig. 4). The elevated right-sided heart pressure was deemed likely due to compression of the RV by the mass, and the elevated PCWP was considered to be mainly caused by LV diastolic dysfunction. The coexistence of broad constrictive pericarditis and restrictive cardiomyopathy had to be taken into consideration. However, CT only showed limited calcification around the mass, which was suggestive of local pericardial involvement. The thickness of the LV wall and the patient's MRI findings ruled out coexisting restrictive cardiomyopathies, such as cardiac amyloidosis and hemochromatosis, but idiopathic restrictive cardiomyopathy was not excluded.

Since we concluded that removing the mass would relieve the symptoms caused by the right-sided HF, surgery was performed. After performing median sternotomy, we found that the mass had adhered to the inner surface of the artificial pericardium created during the previous surgery. After the pericardium was peeled away and resected, we realized that the mass was an organized hematoma. It had also adhered to the epicardium of the RV and the RV outflow tract patch, but the area of pericardial adhesion around the hematoma was limited. We did not detect any large feeding arteries. We tried to resect as much of the hematoma and the surrounding granulation tissue as we could, but some of its 


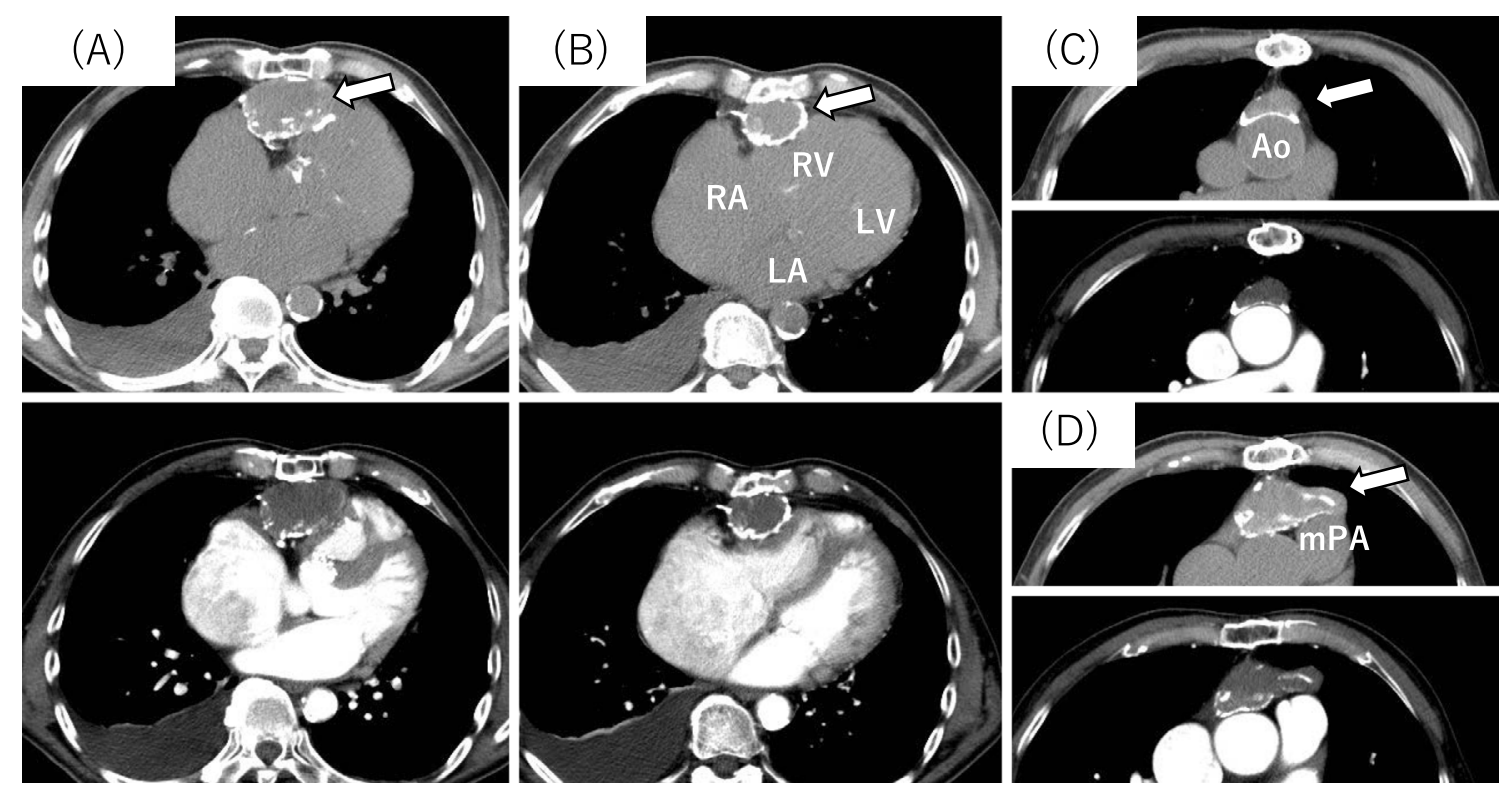

Figure 2. Plain (upper) and contrast-enhanced (lower) computed tomographic scan images. The arrow indicates the hematoma with its calcified capsule located behind the sternum. It had compressed the right ventricle (A, B) and extended to the anterior walls of the ascending aorta (C) and main pulmonary artery (D). Ao: aorta, mPA: main pulmonary artery, RA: right atrium, RV: right ventricle, $L A$ : left atrium, $L V$ : left ventricle
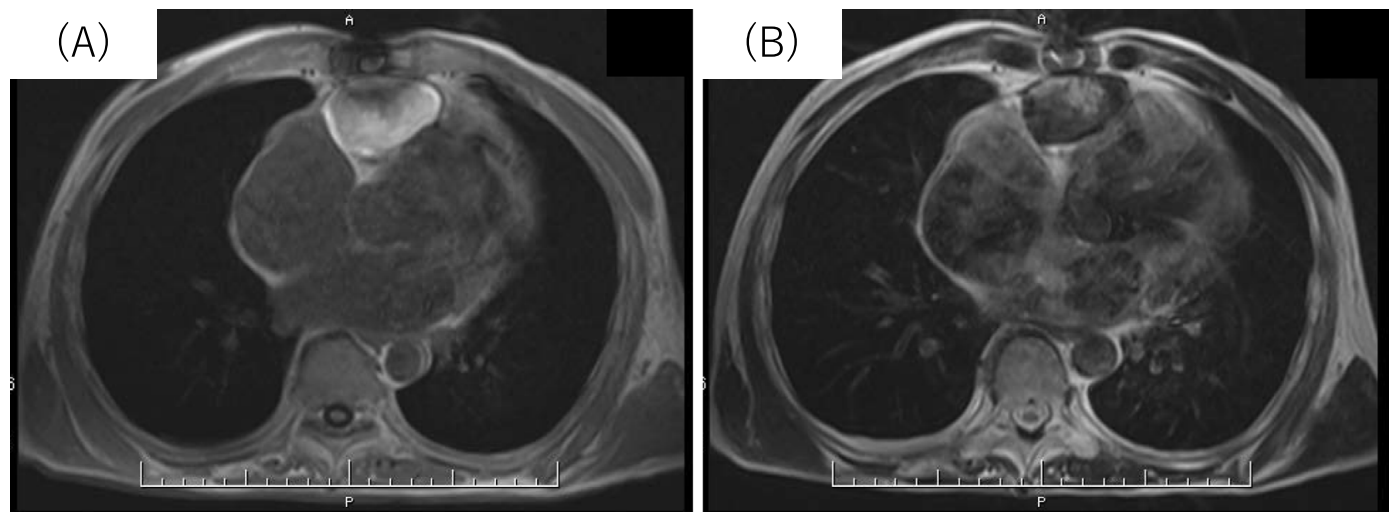

Figure 3. Cardiac magnetic resonance imaging findings. T1-weighted images showed a high-intensity area surrounded by a low-intensity rim (A). T2-weighted images showed a mixture of non-homogeneous low- and high-intensity areas surrounded by a low-intensity rim (B).

capsule remained on the patch. The patient's postoperative course was uneventful. His edema soon disappeared, and the diuretic dose was tapered before finally being discontinued. No recurrence of the hematoma was observed at over four years after surgery. The patient's HF was so well controlled that we were able to rule out coexisting idiopathic restrictive cardiomyopathy.

\section{Discussion}

Generally, hematomas in tissues or organs resolve within a short period of time if there is no continuous bleeding or infection, but $\mathrm{CEH}$ can develop into large masses over long periods of time. The precise mechanism responsible for the development of $\mathrm{CEH}$ is unclear, but a similar mechanism is considered to be involved in the development of chronic subdural hematomas (5). A steep osmotic pressure gradient is produced by the breakdown products of hematomas, resulting in localized inflammatory reactions. Factors in the coagulation cascade along with the release of vasoactive substances are considered to be associated with inflammatory reactions, which might cause additional bleeding from fragile capillaries, and such bleeding can lead to further inflammation in a cycle that can result in the development of a hematoma.

CEIHs are very rare, but their precise incidence is not known. After the causative injuries, most CEIHs seem to develop within a short period of time (three weeks to one year). However, some CEIHs develop over several years. A thorough search of the literature only revealed 10 cases of 
CEIH that developed slowly (more than one year) after open-heart surgery $(2,6-14)$ (Table). In addition to our case, another six cases from Japan were reported. Nine cases involved men, and one involved a woman. Most patients were $>60$ years old. The causative surgery was coronary artery bypass grafting in five cases and valve replacement in four cases. One case developed after the repair of a double-outlet right ventricle (DORV). In most cases, solitary CEIHs were found, but two hematomas were detected in one case. Most hematomas were $>40 \mathrm{~mm}$ in diameter and located adjacent to the left atrium, left ventricle, or both. It was considered that these chambers were affected because the causative surgery injured them or the surrounding tissues. Most patients

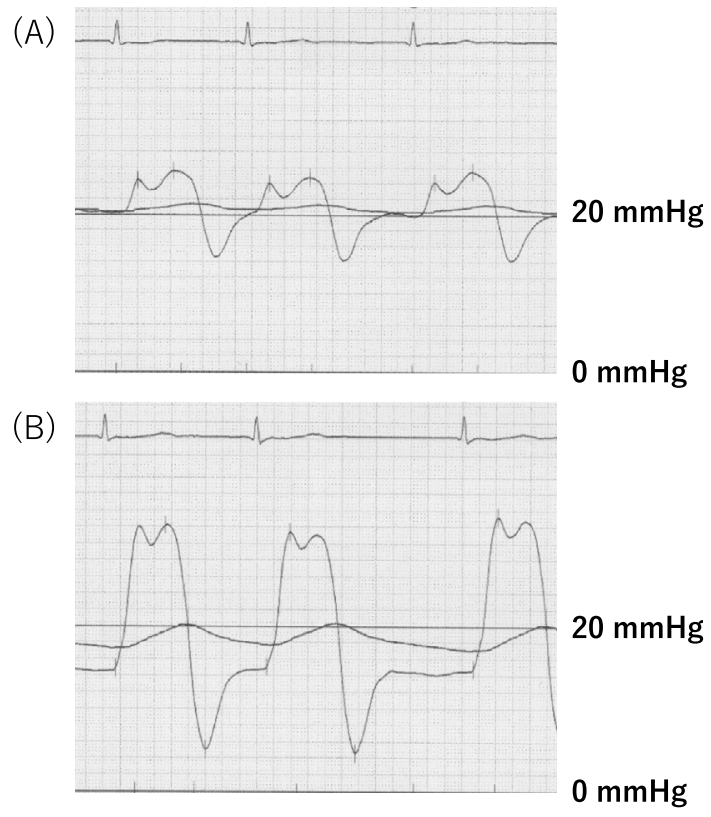

Figure 4. Right atrium (A) and right ventricle (B) pressure. Tracing of the right atrium pressure demonstrated a rapid and deep y descent, and that of the ventricle pressure demonstrated an abnormal filling pattern. presented with HF due to diastolic disturbance in the affected chambers. In our case, since the previous TOF repair procedure involved the closure of a ventricular septal defect and opening of the RV outflow tract, the hematoma developed anterior to the RV, compressing it, which caused rightsided HF. It had probably gradually increased in size for three years, resulting in it becoming refractory to medical treatment. Oral anticoagulant treatment seemed to modify the development of the hematoma. In the case involving a patient with a DORV reported by Hirai et al., (4) a hematoma developed on the left side of the heart, but the surgical procedures performed and the reasons why the hematoma appeared were unclear. As DORV repair can be performed on the ascending aorta and coronary arteries, hematomas might appear on the left side of the heart.

The duration of the period from the causative surgery to the removal of the hematoma varied. In most cases, the hematomas were removed within 10 years, while in our case, the patient's symptoms slowly developed over 27 years, and the hematoma was finally removed after 30 years. In the previous case involving the repair of a DORV, the hematoma was removed at 14 years after the surgery. Interestingly, in two cases involving congenital heart disease, the duration between the original surgery and the removal of the hematomas were long, but the reasons for this long duration were unclear.

TTE is non-invasive and easy to perform and can be used to differentiate intracardiac hematomas from all other conditions. However, it is important to recognize that there are a few blind spots that cannot be viewed with this imaging modality. The hematoma in our case was located behind the sternum and thus was not detected on serial CXR or TTE. CT can be used to detect, localize, and characterize hematomas. Such characterization is best accomplished when both plain and contrast-enhanced images are obtained. Furthermore, CT can be used to differentiate intracardiac thrombi from intrapericardial hematomas. This modality is

Table. Clinical Description of CEIHs Developed over More than One Year after Open-heart Surgery.

\begin{tabular}{|c|c|c|c|c|c|c|c|c|c|}
\hline $\begin{array}{c}\text { Reference } \\
\text { number }\end{array}$ & Country & Age & Sex & $\begin{array}{l}\text { Type of } \\
\text { surgery }\end{array}$ & $\begin{array}{l}\text { Number of } \\
\text { hematoma }\end{array}$ & $\begin{array}{l}\text { Cardiac } \\
\text { chamber } \\
\text { affected }\end{array}$ & Chief symptom & $\begin{array}{c}\text { Size of } \\
\text { hematoma } \\
(\mathrm{mm})\end{array}$ & $\begin{array}{c}\text { Duration from } \\
\text { surgery to } \\
\text { removal }\end{array}$ \\
\hline 2 & USA & 35 & M & $\mathrm{AVR}+\mathrm{MVR}$ & 1 & RA & Left-sided HF & Not described & 31 months \\
\hline 6 & Japan & 63 & M & AVR & 2 & LA, LV & Left-sided HF & Not described & 8 years \\
\hline 7 & Japan & 63 & $\mathrm{~F}$ & DORV repair & 1 & LA, LV & Left-sided HF & $88 \times 66$ & 14 years \\
\hline 8 & Japan & 85 & M & CABG & 1 & LV & Left-sided HF & $47 \times 24$ & 5 years \\
\hline 9 & Germany & 71 & M & CABG & 1 & LV & Chest pain & $70 \times 40 *$ & 10 years \\
\hline 10 & New Zealand & 78 & M & CABG & 1 & RV & Right-sided HF & $80 \times 50$ & 3 years \\
\hline 11 & Japan & 74 & M & $\begin{array}{c}\mathrm{CABG}+\mathrm{LV} \\
\text { thrombectomy }\end{array}$ & 1 & $\mathrm{RV}$ & Right-sided HF & $40 \times 15^{*}$ & 1 year \\
\hline 12 & India & 61 & M & MVR & 1 & LV & Left-sided HF & $65.4 \times 31.2$ & 20 months \\
\hline 13 & Japan & 68 & M & $\begin{array}{l}\text { MVP+Maze } \\
\text { procedure }\end{array}$ & 1 & LV & Left-sided HF & $71 \times 39^{*}$ & 3 years \\
\hline 14 & Japan & 55 & M & CABG & 1 & RV & Right-sided HF & $50 \times 60$ & 8 years \\
\hline
\end{tabular}

* Size was measured from the figures in the prints. AVR: aortic valve replacement, MVR: mitral valve replacement, DORV: double-outlet right ventricle, CABG: coronary artery bypass grafting, LV: left ventricle, LA: left atrium, RV: right ventricle, RA: right ventricle, HR: heart failure 
also useful for differentiating intrapericardial hematomas from other soft tissue tumors. MRI is useful for further characterizing pericardial masses (15). The precise localization of the mass, whether the mass has infiltrated surrounding structures, the presence/absence of pericardial and/or pleural effusion, and the signal intensity and degree of delayed enhancement on cine MRI can all help distinguish different types of pericardial masses. In our case, the hematoma was finally detected on CT. When a patient who has suffered a heart injury complains of any symptoms related to heart disorders, CT should be performed, even if the injury occurred a long time ago.

To treat CEIH, complete resection of the hematoma, including the capsule and feeding vessel, is recommended because a CEIH recurred in a previous case (10). However, this can sometimes be difficult to achieve, as CEIH can adhere tightly to tissues. Therefore, in our case, careful followup will be required.

\section{Conclusion}

We experienced a rare case of $\mathrm{CEIH}$ in a patient who was suffering from refractory right-sided HF. The CEIH was identified and removed 30 years after surgery to repair TOF. The hematoma was not detected on TTE or CXR since it was located just behind the sternum; however, CT revealed it. If a patient with a prior heart injury complains of heart symptoms, CT should be performed.

The authors state that they have no Conflict of Interest (COI).

\section{References}

1. Reid JD, Kommareddi S, Lankerani M, Park MC. Chronic expanding hematomas. A clinicopathologic entity. JAMA 244: 24412442, 1980

2. Grishkin BA, Catalano PW, Watts MA. Isolated right atrial compression as a late sequela of aortic valve replacement. Tex Heart Inst J 21: 225-227, 1994.

3. Hartl WH, Kreuzer E, Reuschel-Janetschek E, Schmidt D, Reichart B. Pericardial mass mimicking constrictive pericarditis.
Ann Thorac Surg 52: 557-559, 1991.

4. Sughiura $T$, Nishida $H$, Ishitoya $H$, et al. Chronic expanding intrapericardial hematoma after pericardial paracentesis. J Card Surg 21: 491-493, 2006.

5. Labadie EL, Glover D. Physiopathogenesis of subdural haematomas. Part 1. Histological and biochemical comparisons of subcutaneous haematoma in rats with subdural haematoma in man. $\mathrm{J}$ Neurosurg 45: 382-392, 1976.

6. Kagaya S, Abe T, Sekine S, et al. Intrapericardial organized hematoma; a rare complication after open heart surgery. J Thorac Cardiovasc Surg 47: 291-294, 1999.

7. Hirai S, Hamanaka Y, Mitsui N, Isaka M, Kobayashi T. Chronic expanding hematoma in the pericardial cavity after cardiac surgery. Ann Thorac Surg 75: 1629-1631, 2003.

8. Kainuma S, Masai T, Yamauchi T, et al. Chronic expanding intrapericardial hematoma after coronary artery bypass surgery presenting with congestive heart failure. Ann Thorac Cardiovasc Surg 14: 52-54, 2008.

9. Wechsel M, Ropers D, Ropers U, et al. Organized intrapericardial hematoma after coronary artery bypass surgery. J Cardiovasc Comput Tomogr 2: 328-331, 2008.

10. Singhal P, Riordan J. Intrapericardial haematoma: a rare cause of congestive cardiac failure after three years of coronary artery bypass grafting. Heart Lung Circ 17: 343-344, 2008.

11. Okamoto $Y$, Matsumoto $M$, Inoue $H$. Cystic mass formation in constrictive pericarditis and epicarditis after open-heart surgery. Interact Cardiovasc Thorac Surg 8: 589-591, 2009.

12. Basha KM, Palanisamy V, Janakiraman E, et al. A rare presentation of intrapericardial hematoma 20 months post aortic valve replacement. Indian Heart J 67: 148-151, 2015.

13. Nakae M, Mizoguchi H, Yoshitatsu M, Toda K, Sawa Y. Successful surgical case of refractory chronic expanding intrapericardial hematoma treated with preoperative coil embolization of the feeding vessels. Clin Case Rep 7: 1526-1528, 2019.

14. Yamaguchi $T$, Terashima $M$, Takamura $C$, et al. Cardiac magnetic resonance imaging of very late intrapericardial hematoma 8 years after coronary artery bypass grafting. Intern Med 57: 975-978, 2018.

15. Ariyarajah V, Jassal DS, Kirkpatrick I, Kwong RY. The utility of cardiovascular magnetic resonance in constrictive pericardial disease. Cardiol Rev 17: 1-6, 2009.

The Internal Medicine is an Open Access journal distributed under the Creative Commons Attribution-NonCommercial-NoDerivatives 4.0 International License. To view the details of this license, please visit (https://creativecommons.org/licenses/ by-nc-nd/4.0/).

(C) 2021 The Japanese Society of Internal Medicine

Intern Med 60: 2627-2631, 2021 